\title{
WAVE PROPAGATION NEAR A SMOOTH CAUSTIC1
}

\author{
BY DONALD LUDWIG
}

\author{
Communicated by W. Rudin, May 5, 1965
}

A caustic is an envelope of a family of rays of geometrical optics. At a caustic, the usual equations of geometrical optics are not valid. We give a formal asymptotic series, valid both near and away from a smooth caustic, which satisfies the differential equation exactly. In the case of high-frequency oscillations, the solution is represented in terms of the Airy function and its derivative. There is a more general formulation (corresponding to a progressing wave expansion) in which the solution is expanded in terms of solutions of the Tricomi equation. Our procedure can be applied to general linear hyperbolic or time-reduced partial differential equations. The coefficients in our expansion are nearly the same as the coefficients which appear in ordinary geometrical optics; the only essential difference is that a factor which is singular at the caustic has been removed. Our terminology is explained in R. Courant, Methods of mathematical physics, Vol. II, Ch. VI.

In order to illustrate our procedure, we consider the reduced wave equation $\Delta u+k^{2} u=0$, and we give only the leading term in the expansion. All of the essential features are illustrated in this special problem. We write the first term as

(1) $u(x)=\exp (i k \theta(x))\left[A\left(-k^{2 / 8} \rho(x)\right) g(x)+\frac{i}{k^{1 / 8}} A^{\prime}\left(-k^{2 / 8} \rho(x)\right) h(x)\right]$.

Here $A$ denotes the Airy function; we have

$$
A^{\prime \prime}(t)=t A(t)
$$

and

$$
A^{\prime \prime \prime}(t)=t A^{\prime}+A(t) .
$$

The functions $\theta, \rho, g$ and $h$ are determined below. The caustic will be obtained by setting $\rho(x)=0$. Applying the differential operator, using (2) and (3) and collecting terms, we obtain

1 The research in this paper was supported by the National Science Foundation under grant No. GP-3668. 


$$
\begin{aligned}
e^{-i k \theta}\left(\Delta u+k^{2} u\right)= & {\left[k^{2} A g+i k^{5 / 3} A^{\prime} h\right]\left[1-(\nabla \theta)^{2}-\rho(\nabla \rho)^{2}\right] } \\
& +\left[-2 i k^{5 / 3} A^{\prime} g+2 k^{4 / 3} A^{\prime \prime} h\right][\nabla \theta \cdot \nabla \rho] \\
& +i k A\left[2 \nabla \theta \cdot \nabla g+\Delta \theta g+2 \rho \nabla \rho \cdot \nabla h+\rho \Delta \rho h+(\nabla \rho)^{2} h\right] \\
& -k^{2 / 3} A^{\prime}[2 \nabla \rho \cdot \nabla g+\Delta \rho g+2 \nabla \theta \cdot \nabla h+\Delta \theta h] \\
& +A \Delta g+\frac{i}{k^{1 / 3}} A^{\prime} \Delta h .
\end{aligned}
$$

The terms of highest order vanish if we set

$$
\begin{aligned}
1-(\nabla \theta)^{2}-\rho(\nabla \rho)^{2} & =0, \\
\nabla \theta \cdot \nabla \rho & =0 .
\end{aligned}
$$

As we shall see, equations (4) and (5) are equivalent to the eikonal equation. The terms of next lowest order vanish if we set

$$
\begin{gathered}
2 \nabla \theta \cdot \nabla g+\Delta \theta g+2 \rho \nabla \rho \cdot \nabla h+\rho \Delta \rho h+(\nabla \rho)^{2} h=0, \\
2 \nabla \rho \cdot \nabla g+\Delta \rho g+2 \nabla \theta \cdot \nabla h+\Delta \theta h=0 .
\end{gathered}
$$

The remainder $A \Delta g+\left(i A^{\prime} / k^{1 / 3}\right) \Delta h$ can be absorbed into equations analogous to (6) and (7) for the terms of next highest order, and so forth.

In order to satisfy (4) and (5), let $\phi_{+}$and $\phi_{-}$be the phases given by geometrical optics near the caustic. They satisfy $\left(\nabla \phi_{ \pm}\right)^{2}=1$. We define $\theta, \psi$, and $\rho$ by

$$
\begin{aligned}
& \theta(x)=\frac{1}{2}\left(\phi_{+}+\phi_{-}\right), \\
& \psi(x)=\frac{1}{2}\left(\phi_{+}-\phi_{-}\right), \\
& \rho(x)=\left(\frac{3}{2} \psi\right)^{2 / 3} .
\end{aligned}
$$

It follows that $\theta$ and $\rho$ satisfy (4) and (5). The caustic, where $\phi_{+}=\phi_{-}$, is obtained by setting $\rho(x)=0$. It can be shown that if the caustic is smooth, $\theta(x)$ and $\rho(x)$ are regular functions of $x$. Equations (4) and (5) can also be solved directly by the method of characteristics. The characteristics of the system (4), (5) are the rays of geometrical optics.

In order to compare our equations (6) and (7) with the transport equation of geometrical optics, we define

$$
a_{ \pm}=g \pm \sqrt{ } \rho h .
$$

Forming the sum of (6) $\pm \sqrt{ } \rho$ (7), we obtain

$$
2(\nabla \theta \pm \sqrt{ } \rho \nabla \rho) \cdot \nabla a_{ \pm}+(\Delta \theta \pm \sqrt{ } \rho \Delta \rho) a_{ \pm}=0,
$$

or, using (8)-(10), 


$$
2 \nabla \phi_{ \pm} \cdot \nabla a_{ \pm}+\left(\Delta \phi_{ \pm} \mp \frac{(\nabla \psi)^{2}}{3 \psi}\right) a_{ \pm}=0 .
$$

Equation (13) differs from the transport equation of geometrical optics only in the term $\pm(\nabla \psi)^{2} / 3 \psi$. Thus $a_{ \pm}=\rho^{1 / 4} b_{ \pm}$, where $b_{ \pm}$is the amplitude given by geometrical optics. This additional factor makes $a_{ \pm}$bounded at the caustic.

To complete the identification of our approximate solution with geometrical optics, using (11) and the asymptotic expansion of the Airy function for $k^{2 / 3} \rho \gg 0$, if $\rho>0$, we obtain

$$
u \sim \frac{-i}{2 \sqrt{ } \pi k^{1 / 6} \rho^{1 / 4}}\left[\exp \left(i k \phi_{+}+\frac{i \pi}{4}\right) a_{+}-\exp \left(i k \phi_{-}-\frac{i \pi}{4}\right) a_{-}\right] .
$$

Thus, on the illuminated side of the caustic, our approximation coincides with the one given by geometrical optics. The outgoing $(+)$ and incoming $(-)$ parts of (14) are connected by the requirement, from (11), that $a_{+}=a_{-}$at the caustic. The presence of the terms $\pm i \pi / 4$ in the exponents of (14) gives rise to the well-known phase shift of $\pi / 2$ after a wave passes through the caustic.

It can be seen from (12) that $a_{+}$and $a_{-}$are regular functions of $\theta$ and $\sqrt{ } \rho$, which implies that $g$ and $h$ are regular functions of $x$. If the caustic is analytic, the functions $\theta, \rho, g$ and $h$ may be extended to the shaded region, where $\rho<0$; in this case the asymptotic behavior of the Airy function for $-k^{2 / 3} \rho \gg 0$ shows that our solution has an exponential decay given by $\exp \left(-k \frac{2}{3}(-\rho)^{3 / 2}\right)$. We may conjecture a similar result in the neighborhood of a nonanalytic caustic.

A more general expansion is obtained in the form

$$
u(x)=\sum_{j=0}^{\infty}\left[U_{\theta}^{j}(\theta, \rho) g^{j}+U_{\rho}^{j}(\theta, \rho) h^{j}\right],
$$

where $\partial U^{i+1} / \partial \theta=U^{i}$, and

$$
U_{\rho \rho}^{j}(\theta, \rho)=\rho U_{\theta \theta}^{j}(\theta, \rho),
$$

i.e., $U^{i}$ satisfies the Tricomi equation. The calculations are virtually identical with the preceding ones. The more general expansion enables us to solve problems involving propagation of discontinuities or other singularities in space-time.

Asymptotic expansions near a smooth caustic, which are similar to ours, have been obtained by a boundary layer technique by $R$. Buchal and J. B. Keller [2] and R. Buchal [1]. The present work was partly motivated by B. Friedman [3] and F. W. J. Olver [4]. 
I would like to thank R. M. Lewis, J. B. Keller and N. Bleistein for many helpful conversations on this subject.

\section{REFERENCES}

1. R. Buchal, The boundary layer formalism for convergent series solutions of the wave equation with reference to the focussing of discontinuities. Parts I and II, Univ. of Wisconsin, MRC Tech. Rep. No. 199 and No. 211, 1960 and 1961.

2. R. Buchal and J. B. Keller, Boundary layer problems in diffraction theory, Comm. Pure Appl. Math. 13 (1960), 85-114.

3. B. Friedman, Stationary phase with neighboring critical points, J. Soc. Indust. Pure Appl. Math. 13 (1960).

3. B. Friedman, Stationary phase with neighboring critical points, J. Soc. Ind. Appl. Math. 7 (1959), 280-289.

4. F. W. J. Olver, The asymptotic solution of linear differential equations of the second order for large values of a parameter, Philos. Trans. Roy. Soc. London Ser. A 247 (1954), 307-327.

NEW YORK UNIVERSITY 